\title{
Managing DICOM images: Tips and tricks for the radiologist
}

\author{
Dandu Ravi Varma \\ Department of Radiology, Krishna Institute of Medical Sciences, Minister Road, Hyderabad, India \\ Correspondence: Dr. D. Ravi Varma, Department of Radiology, Krishna Institute of Medical Sciences, Minister Road, Hyderabad, India. \\ E-mail: varmaji@rediffmail.com
}

\begin{abstract}
All modalities in radiology practice have become digital, and therefore deal with DICOM images. Image files that are compliant with part 10 of the DICOM standard are generally referred to as "DICOM format files" or simply "DICOM files" and are represented as ".dcm." DICOM differs from other image formats in that it groups information into data sets. A DICOM file consists of a header and image data sets packed into a single file. The information within the header is organized as a constant and standardized series of tags. By extracting data from these tags one can access important information regarding the patient demographics, study parameters, etc. In the interest of patient confidentiality, all information that can be used to identify the patient should be removed before DICOM images are transmitted over a network for educational or other purposes. In addition to the DICOM format, the radiologist routinely encounters images of several file formats such as JPEG, TIFF, GIF, and PNG. Each format has its own unique advantages and disadvantages, which must be taken into consideration when images are archived, used in teaching files, or submitted for publication. Knowledge about these formats and their attributes, such as image resolution, image compression, and image metadata, helps the radiologist in optimizing the archival, organization, and display of images. This article aims to increase the awareness among radiologists regarding DICOM and other image file formats encountered in clinical practice. It also suggests several tips and tricks that can be used by the radiologist so that the digital potential of these images can be fully utilized for maximization of workflow in the radiology practice.
\end{abstract}

Key words: Compression; DICOM; image file; management; PowerPoint ${ }^{\circledR}$; resolution

\section{Introduction}

As radiologists we deal with DICOM (digital imaging and communications in medicine) image files sourced from different modalities, either in a standalone or integrated manner. DICOM files have several unique features, the knowledge of which is important for the practicing radiologist. This article aims to increase the awareness of radiologists regarding DICOM and other image files so that all their features can be fully exploited.

\begin{tabular}{|l|l|}
\hline \multicolumn{2}{|c|}{ Access this article online } \\
\hline Quick Response Code: & \\
\hline & Website: \\
\hline & www.iji.org \\
\cline { 2 - 2 } & DOI: \\
\hline
\end{tabular}

\section{What is a DICOM Image File?}

The DICOM standard is useful for integrating all modern imaging equipments, accessories, networking servers, workstations, printers, and picture archiving and communication systems (PACS) that may have been installed by multiple manufacturers. ${ }^{[1]}$ Because of its ease of integration and continuous evolution this communication standard has over the years achieved a nearly universal level of acceptance among vendors of radiological equipment.

A DICOM image file is an outcome of the Digital Imaging and Communications in Medicine standard. Specifically, image files that are compliant with part 10 of the DICOM standard are generally referred to as "DICOM format files" or simply "DICOM files" and are represented as ".dcm."[2]

\section{Why cannot DICOM Files be Viewed Directly on a Personal Computer?}

There are several situations where DICOM files find their 
way into radiology practice. One common example of this is when information from a radiological study is exported into an offline medium such as a compact disk (CD) for easy transport or archival. Such a CD usually contains several DICOM image files as well as other files that are necessary for display of these images. Even though the specific folder architecture varies from vendor to vendor, the CDs usually contain an autorun file, a DICOM viewer, a DICOM directory (DICOMDIR), and a folder containing the DICOM images [Figure 1].

Inconveniently, in contrast to other image file formats such as JPEG or TIFF files, the individual DICOM files are not recognized by Windows ${ }^{\circledR}$ as image files. As a result, one is not able to view the contents of the image by simply double clicking on them. ${ }^{[3]}$

To view these images on computers when a proprietary viewer is not supplied with the system, an additional software package called "DICOM browser" is needed, which will interpret the file information and display it as an image. A list of free DICOM browsers has been published earlier in this journal. ${ }^{[4]}$

\section{What are the Parts of a DICOM File?}

DICOM differs from other image formats in that it groups information into data sets. ADICOM file consists of a header and image data sets, all packed into a single file [Figure 2].

The first few packets of information in a DICOM image file constitute the "header." It stores demographic information about the patient, acquisition parameters for the imaging study, image dimensions, matrix size, color space, and a host of additional nonintensity information required by the computer to correctly display the image. The header is

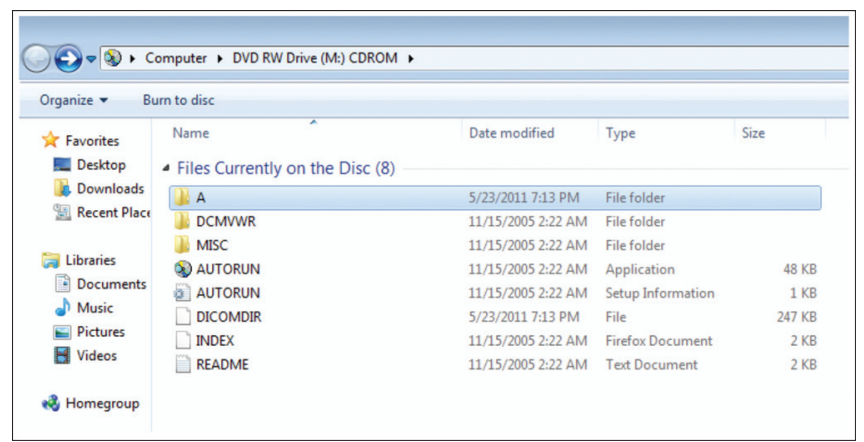

Figure 1: Managing DICOM files in a CD: screenshot of contents of a CD containing an MRI study (prepared on a Advantage Windows Workstation (GE Medical Systems)). Folder "A" contains DICOM image files from the MRI study; folder "DCMVWR" contains the Dicomviewer that displays the contents of the CD; the folder "MISC" contains miscellaneous files required during display; "AUTORUN" files direct the actions that are automatically performed when the $C D$ is introduced into a computer. The DICOMDIR is essentially an index and summary of information regarding all DICOM files included in that CD followed by a single attribute (7FE0) that contains all the pixel intensity data for the image. ${ }^{[5]}$ These data are stored as a long series of $0 \mathrm{~s}$ and $1 \mathrm{~s}$, which can be reconstructed as the image by using the information from the header. This attribute may contain information regarding a single image, multiple frames of a study, or a cine loop, depending on the modality that has generated the image.

The header data information is encoded within the DICOM file so that it cannot be accidentally separated from the image data. If the header is separated from the image data, the computer will not know which imaging study has been done or to whom it belongs and it will not be able to display the image correctly, leading to a potential medicolegal situation.

\section{Preamble (128 bytes)}

$$
\text { Prefix - 'D', 'I', 'C', ‘M' }
$$

\section{Header:}

Data Set

- Group 1 (0002)

- Element $1(0002,0000)$

- Element $2(0002,0001)$

- Element 3...etc.

- Group 2 (0008)

- Group 3...etc.

Image Pixel Intensity Data: 10011010011001011010100 01011010100100110100110 10100110010110101001001 10011010011001011010100 01011010100100110100111 $10100110010110101001 \ldots . .$. 


\section{How can one Read the Header Information?}

The information within the header is organized as a constant and standardized series of tags. These tags are organized into groups of data elements. For example, the group "0010" contains patient information and is 92 bits in length. It contains the patient's name in the tag "0010-0010," the patient's identification number in the tag "0010-0020," birth date in the tag "0010-0030," and so on. Similarly the group "0018" contains information regarding acquisition. It is 482 bits long and contains several elements that convey the MRI acquisition parameters. The group "0028” encodes image presentation and is responsible for display of the image on a monitor $^{[6]}$ [Figure 3].

Analyzing the DICOM header may also give valuable information regarding the imaging study itself. For example, if a radiologist encounters a good-quality MRI image and would like to replicate the MRI pulse sequence on his MRI scanner, he can easily access all relevant parameters from the DICOM header.
There are several freely available software packages that can be used to extract information from the DICOM header. DicomWorks is a popular DICOM viewer that can view header information. ${ }^{[7]}$ Other examples of software that can be used to explore the contents of the header include Image ${ }^{[8]}$ and XnView. ${ }^{[9]}$

\section{How do I Remove Patient Information from DICOM Images?}

The common tags that indicate the patient identity include the patient's name, age, sex, birth date, hospital identity number, ethnic group, occupation, referring physician, institution name, study date, and DICOM Unique Identifiers (UIDs). As described earlier, such demographic information of the patient and a host of other information about the imaging study is encoded within an image header. The data may or may not be displayed on the screen, but the information can be extracted from the header by anyone who has access to the DICOM file. Several educational resources using DICOM files are available for radiology

\begin{tabular}{|c|c|c|c|c|}
\hline \multicolumn{5}{|c|}{ Simple editor Detailed editor } \\
\hline \multicolumn{2}{|c|}{ List only tags COMMON to ALL files } & \multicolumn{2}{|c|}{ - 315 tags displayed (total=315 tags) } & \multirow[b]{2}{*}{ Value } \\
\hline & All existing tags & [Group,Element] & Title & \\
\hline & Variant tags & {$[0010-0000]$} & Patient Group Length & 92 \\
\hline & Critical tags & {$[0010-0010]$} & Patient's Name & xssossx \\
\hline$[0002]$ & File Meta Elements & {$[0010-0020]$} & Patient ID & EX/10/00832 \\
\hline [0008] & Study information & {$[0010-0030]$} & Patient's Bitth Date & 19640101 \\
\hline [0009] & Private & {$[0010-0040]$} & Patient's Sex & \\
\hline [0010] & Patient & [0010-1010] & Patient's Age & $000 Y$ \\
\hline [0011] & Private & [0010-1030] & Patient's Weight & 80 \\
\hline [0018] & Acquisition Group & {$[0010-2180]$} & Additional Patient History & \\
\hline [0019] & Private & {$[0011.0000]$} & group length & 30 \\
\hline [0020] & Relationship Group & {$[0011.0010]$} & Inconnu & GEMS_PATI_01 \\
\hline [0021] & Private & {$[0011 \cdot 1010]$} & Inconnu & Num $=0-\overline{S t}=$ \\
\hline [0023] & Private & {$[0018-0000]$} & Aquisition Group Lenght & 482 \\
\hline [0025] & Private & {$[0018.0020]$} & Scanning Sequence & SE \\
\hline [0027] & Private & {$[0018-0021]$} & Sequence Variant & NONE \\
\hline [0028] & Image presentation & {$[0018.0022]$} & Scan Options & SAT_GEMSIVB_GEMSIFILTERED_GEMSISP \\
\hline [0029] & Private & [0018-0023] & MR Acquisition Type & 20 \\
\hline [0040] & Private & [0018-0025] & Angio Flag & $\mathrm{N}$ \\
\hline [0043] & Private & [0018-0050] & Slice Thickness & 5 \\
\hline [7FEO] & Pixel Data & [0018-0080] & Repetition Time & 3000 \\
\hline & Tags to modify & [0018-0081] & Echo Time & 92.976 \\
\hline & Tags to add & {$[0018.0082]$} & Inversion Time & 0 \\
\hline & Tags to remove & [0018-0083] & Number of Averages & 2 \\
\hline & Search & [0018-0084] & Imaging Frequency & 63.867787 \\
\hline & & [0018-0085] & Imaged Nucleus & $1 \mathrm{H}$ \\
\hline & & [0018-0086] & Echo Numbers & 1 \\
\hline & & {$[0018-0087]$} & Magnetic Field Strength & 1.5 \\
\hline & & [0018-0088] & Spacing Between Slices & 6.5 \\
\hline & & [0018-0091] & Echo Train Length & 16 \\
\hline & & [0018-0093] & Percent Sampling & 100 \\
\hline & & {$[0018.0094]$} & Percent Phase Field of View & 75 \\
\hline & & [0018-0095] & Pixel Bandwidth & 61.0547 \\
\hline & & [0018-1000] & Device Serial Number & O00000000000GEHC \\
\hline & & [0018-1020] & Software Versions & 11VXMMR Software release:11.1_M4_0818.a \\
\hline & & [0018-1030] & Protocol Name & BRAIN PLUS/ \\
\hline & & [0018-1088] & Heart Rate & 0 \\
\hline & & [0018-1090] & Cardiac Number of Images & 0 \\
\hline & & [0018-1094] & Trigger Window & 0 \\
\hline & & [0018-1100] & Reconstruction Diameter & 240 \\
\hline & & [0018-1250] & Receiving Coil & SNVHEAD NECK_A \\
\hline & & {$[0018-1310]$} & Acquisition Matrix & @I \\
\hline & & [0018-1312] & Phase Encoding Direction & ROW \\
\hline & & [0018-1314] & Flip Angle & 90 \\
\hline & & [0018-1315] & Variable Flip Angle Flag & $\mathrm{N}$ \\
\hline & & 10018-1316i & SAR & 1.642500 \\
\hline
\end{tabular}

Figure 3: Screenshot of the DICOM tags extracted from an MRI image by Dicomworks, a popular DICOM viewer software. Note the rich variety of information regarding the patient and the imaging study that can be visualized by analyzing the header 
students on the World Wide Web. Creating and accessing such electronic teaching files often involve transmission of DICOM data over the Internet. In the interest of patient confidentiality, all information identifying the patient should be removed from the DICOM header when a DICOM file is uploaded for such purposes.

Respecting the patient's privacy is important when images are used in presentations, teaching files, or publications. A simple and easy method of ensuring this is by converting and exporting the DICOM file into other image formats such as JPEG or TIFF. The header information is lost and patient identity cannot be obtained from the resultant image. Another method is "anonymization," whereby all patient information is removed from the DICOM header. ${ }^{[3]}$ This is achieved by using software like DicomWorks, ImageJ, and FP Image. ${ }^{[7,8,10]}$ Specifically, all tags contained in groups "0008" (study information) and "0010" (patient information) of the DICOM header should be removed and replaced during anonymization.

\section{What are the Other Commonly Used File Formats and when do I need them?}

Although DICOM images have found wide acceptance in medical practice, they have two disadvantages: file sizes are large and special software is required for viewing them on personal computers. Outside the radiology department, most personal computers run on the Windows ${ }^{\circledR}$ operating system, which does not recognize the DICOM file structure. Thus, for incorporating images in PowerPoint ${ }^{\circledR}$ presentations, for creating teaching files, or for publishing in Web pages, DICOM images need to be converted into image formats that can be recognized by Windows ${ }^{\circledR}$.

There are over a hundred formats described to store images, most of them being proprietary. ${ }^{[11]}$ The more popular formats used in daily practice are the JPEG, JPEG 2000, TIFF, GIF, and PNG formats. In contrast to DICOM images, images saved in these formats can be viewed on any personal computer without the need for dedicated viewers. They can be easily incorporated into presentations and Web pages. Image files saved in these formats are devoid of bulky header information and usually contain 8-bit information. These files therefore require less storage space and demand less resource to transfer over a network or via the Internet. One big disadvantage of these file formats, compared to DICOM, is that they contain a user-determined window level and window width that is set at the time of creation of the image.$^{[12]}$ Consequently, the contrast between structures within the image cannot be adjusted and postprocessing cannot be performed on these images.

a. JPEG (Joint Photographic Experts Group): The JPEG format is the most popular format and can be read by all computer platforms. Because JPEG files are small in size and extremely portable, they are the preferred format when transferring images over the Web. The advantage of the JPEG format is that it facilitates use of compression to reduce file size. Typically, the least noticeable bits of information are removed by complex mathematical algorithms, so that the image is represented with less information. When saving as a JPEG file, options are available for selecting the amount of compression that can be applied. The more the file is compressed (lossy compression), the more the original image information lost; such an image will not look good when reproduced. Lesser degrees of compression (lossless compression) retain high image quality, but this is achieved at the cost of a large file size.

b. TIFF (Tagged Image File Format): The TIFF format is versatile and supports the full range of image sizes, resolutions, and color depths. Since TIFF images are saved without compression or with a lossless compression scheme they retain the original image quality and often are large in size. ${ }^{[13]}$ TIFF is preferred where high image quality is desired, for example, when the image contains illustrations and line diagrams.

c. GIF (Graphics Interchange Format): GIF is an old file format that is compatible with older versions of internet browsers and other software. A major advantage of this format is its ability to save animations. It can store only a limited amount of color information and is becoming increasingly unpopular as a format for storing digital images.

d. PNG (Portable Networks Graphics): The PNG file format was developed to outperform and eventually replace the GIF format. It has better browser compatibility and supports greater color depth than the GIF format. Its lossless compression enables better image quality, though at the expense of large file sizes. ${ }^{[13]}$

Which of these file types is ideal for radiology practice? Generally speaking, lossy files are ideal for use in computer and Web-based presentations, where the small file size permits rapid image display and facilitates easy image transfer between computers. In contrast, lossless formats are better when higher image quality is desired, such as for archiving, teaching, and submission for publication [Table 1].

\section{How can I Convert DICOM Images into Other File Formats?}

Conversion of DICOM images into other formats is most often performed at a diagnostic workstation or at a Web client of a PACS system. Most of these systems have an "export" function that enables the operator to save the image displayed in the active window as a JPEG or TIFF file. While some applications permit export of a batch of DICOM images into other formats, most require repeated operations, converting one image at a time. 
Even if the DICOM viewer lacks an image export function, users of the Windows ${ }^{\circledast}$ operating system can press the "Print Screen" key on the keyboard to capture the current monitor display and save by directly pasting it within a PowerPoint ${ }^{\mathrm{TM}}$ slide or by saving it as a file using an image editing software package. Also, the contents of an active window can be selectively captured by pressing "Print Screen" key along with the "Alt" key.

As has been noted earlier, the TIFF format should be preferred when creating a master copy from DICOM files. Though TIFF files have large file sizes, this format provides the highest image quality. Subsequently, the images can be saved in other formats such as JPEG in order to save on storage space. It should be remembered that while it is possible to convert a TIFF image into a low-quality JPEG image, it is not possible to regain the original detail from the JPEG image.

Outside the radiology environment, DICOM files can be exported to other image formats using stand-alone DICOM viewers. Several free stand-alone DICOM browsers (such as DicomWorks, ImageJn and MEDISP Viewer) available on the Internet are good examples of such software. ${ }^{[7,8,14]}$ An earlier article in this series has reviewed the capabilities and limitations of free DICOM browsers. ${ }^{[4]}$

Several image management software packages that permit easy screen capture (such as IrfanView and XnView) are available for free download. For example, it is possible to activate a "capture/screenshot" function in IrfanView that automatically saves the monitor display as an image file when a specified hot key is pressed. The part of the display saved, the hot key, as well as the output file format can be flexibly specified by the user. IrfanView and XnView also have DICOM plug-ins that enable direct viewing of DICOM

Table 1: Comparison and applications of different image file formats

\begin{tabular}{|c|c|c|c|}
\hline $\begin{array}{l}\text { File } \\
\text { format }\end{array}$ & Advantages & Disadvantages & $\begin{array}{l}\text { Applications in } \\
\text { clinical radiology }\end{array}$ \\
\hline JPEG (.jpg) & $\begin{array}{l}\text { Permits setting } \\
\text { compression level; } \\
\text { small image size }\end{array}$ & $\begin{array}{l}\text { Low image quality } \\
\text { at higher degrees of } \\
\text { compression }\end{array}$ & 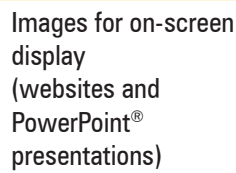 \\
\hline GIF (.gif) & $\begin{array}{l}\text { Good internet } \\
\text { browser support; } \\
\text { good for displaying } \\
\text { animations }\end{array}$ & $\begin{array}{l}\text { Larger file sizes; } \\
\text { limited color support } \\
\text { ( } 256 \text { colors or } 256 \\
\text { shades of gray) }\end{array}$ & $\begin{array}{l}\text { Images for display on } \\
\text { a website }\end{array}$ \\
\hline PNG (.png) & $\begin{array}{l}\text { Good internet } \\
\text { browser support; } \\
\text { good image quality }\end{array}$ & $\begin{array}{l}\text { Limited acceptance } \\
\text { with earlier web } \\
\text { browsers }\end{array}$ & $\begin{array}{l}\text { Teaching files; } \\
\text { publication; images } \\
\text { for display on a } \\
\text { website }\end{array}$ \\
\hline TIFF (.tif) & High image quality & Large file size & $\begin{array}{l}\text { Master copies of } \\
\text { teaching file; format } \\
\text { preferred by journals } \\
\text { for publication }\end{array}$ \\
\hline
\end{tabular}

images. It is also possible to export a batch of DICOM images into other image formats, using the "batch processing" or "batch conversion" function in these software packages. ${ }^{[9,15]}$

\section{How do I Convert an Image into one with an Optimal Resolution?}

When a medical image is viewed on the screen what is actually seen is a collection of digital sample points called "pixels." A pixel is the basic unit of a digital image. The resolution of an image is largely dependent upon the total number of pixels contained in the image, which can be derived by multiplying the number of horizontal and vertical pixels within the image. For example, a CT scan image that is composed of $512 \times 512$ pixels has a total of 262,144 pixels (0.25 megapixels), while a computer radiography $(C R)$ image is composed of $2048 \times 2048$ pixels (4 megapixels). ${ }^{[16]}$ Photographs taken using digital cameras are another common source of large image files in current radiological practice. Digital cameras of over 10 megapixel resolutions have become common place today [Table 2].

It is common knowledge that images containing fewer pixels appear to have poor quality, especially when enlarged. On the other hand, an image containing more information needs larger memory capacity for storage and longer time for transmission and display. This need for a good image resolution in radiology practice is best appreciated when working with computed radiography images, digital mammograms, or digital photos. It may sometimes become necessary to save these images with an optimal (lower) resolution to strike a balance between the desired image quality and file size.

The optimal resolution for an image depends on the anticipated use of the image. Typically, computer monitors and digital projectors have a much lower output resolution than laser cameras or printers. If this article is read on a 14 inch laptop with a screen that displays $1388 \times 768$ pixels, the resolution is about 100 pixels per inch (ppi). Similarly, most projectors support displays of $800 \times 600$ or $1024 \times 768$ pixels. PowerPoint ${ }^{\circledR}$ supports a default resolution of up to 96 ppi. ${ }^{[17]}$ Thus, as far as resolution is concerned, the weakest link in the image display chain is the screen or projector

Table 2: Sizes of images produced by digital imaging modalities

\begin{tabular}{lccc}
\hline Modalities & Pixel matrix & Total pixels & Size of images \\
\hline Nuclear medicine & $128 \times 128$ & 16,384 & $0.16 \mathrm{MP}$ \\
MRI & $256 \times 256$ & 65,536 & $0.06 \mathrm{MP}$ \\
USG & $512 \times 512$ & 262,144 & $0.25 \mathrm{MP}$ \\
DSA & $512 \times 512$ & 262,144 & $0.25 \mathrm{MP}$ \\
CT scan & $512 \times 512$ & 262,144 & $0.25 \mathrm{MP}$ \\
CR / DR & $2048 \times 2048$ & $4,194,304$ & $4 \mathrm{MP}$ \\
Digital mammography & $4000 \times 5000$ & $20,000,000$ & $81 \mathrm{MP}$ \\
Digital cameras & User defined & User defined & $3-20 \mathrm{MP}$ \\
\hline
\end{tabular}


system that displays the image. The image quality perceived by the viewer is often limited by the output device used. Even if a large image (for example a $2048 \times 2048$ pixel digital chest radiograph) is incorporated into a PowerPoint ${ }^{\circledR}$ slide, it can be seen at a much lower resolution on the monitor or when projected on a screen ${ }^{[18]}$ [Figure 4].

To minimize the storage space requirements, the image sizes can be "scaled" or "resized" using software programs that add or subtract pixel information using a mathematical process called interpolation. For use in onscreen presentations or Web pages, the image sizes may be reduced to about 1500 and 800 pixels in the horizontal and vertical dimensions (about 1 megapixel) respectively, without any appreciable loss of quality. On the other hand, print media requires much higher resolutions. Most journals recommend 300 ppi for optimal reproduction of photographs or scans and 600 ppi for line diagrams. ${ }^{[18]}$ This also explains why images saved from PowerPoint ${ }^{\circledR}$ presentations are not adequate for publication.

\section{What is "Batch Image Processing?"}

Every now and then we encounter a series of images, all of which need the same set of tasks for optimization. Tasks like enhancement, windowing, or resizing, etc., can be tedious and time consuming, especially when performed one file at a time. For example, imagine incorporating a series of 20 chest radiographs into a PowerPoint ${ }^{\circledR}$ presentation. The repetitive tasks would include resizing each image to fit into a PowerPoint ${ }^{\circledR}$ slide, manually cropping outpatient information, and adjusting the contrast/brightness, and so on. The "batch processing" technique allows multiple image manipulations to be performed on a series of images as a single convenient operation instead of performing them individually.

A variety of repetitive tasks may be accomplished using batch processing. Conversion of a set of images to another format; renaming images using a user-specified scheme;

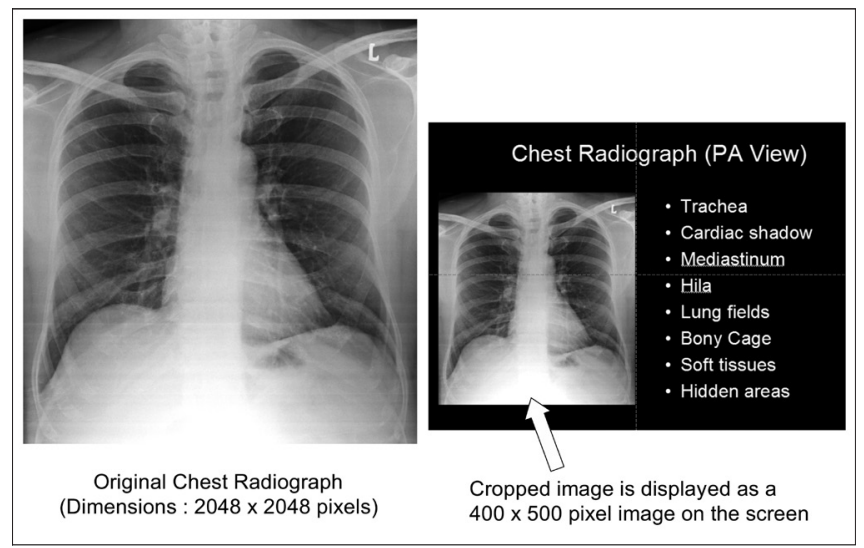

Figure 4: A cropped image incorporated in a PowerPoint slide has low resolution, irrespective of the resolution of the original image converting color images to gray scale; resizing, flipping, or rotating the images; adjusting the resolution and bit depth; cropping the margins; adjusting brightness and contrast; sharpening the image; and adding annotations are all processes often required by radiologists. Imageediting software such as IrfanView and XnView ${ }^{[9,15]}$ enable the operator to perform such batch operations with a single click [Figure 5]. Moreover, these software packages allow the operator to save all the user-defined settings in a file. These settings can be recalled at a later time and the same set of operations can be performed on another series of images.

\section{How do I Compress Images to Save Storage Space?}

One problem radiologists commonly face is storing their growing collections of images. High-field MRI and multidetector CT (MDCT) are generating more and more images of ever increasing resolution daily, contributing significantly to the large volume of digital data. A single image from an MDCT scan of the brain needs approximately the same storage space as a 30-page MS Word ${ }^{\circledR}$ document that contains 18,000 words.

Unfortunately, a huge chunk of storage space is wasted in storing nonessential components of the images. For example, a radiologist will instantaneously recognize Figure 6 as a T1W axial image of the brain. He/she is able to do this simply by analyzing only the pixels in the central $50 \%$ of the image, paying little attention to the black pixels surrounding the brain, which do not contain any diagnostic information. A computer, on the other hand, cannot make this distinction and gives equal importance to all pixels, thereby doubling the storage space requirement. Reduction in file sizes beneficially permits storage of more images in a given amount of disk or memory space. It also reduces the time required for images to be sent over the Internet or downloaded from Web pages.

There are several means available to reduce image file sizes. Storing images in formats other than DICOM can result in significant reduction of storage space requirements. Similar reduction can be achieved by lowering the resolution and cropping unwanted portions of the images with batch processing. Furthermore, converting images acquired in color to gray scale can help, since color images occupy up to four times as much space as the corresponding gray-scale images. ${ }^{[19]}$

Compression is another essential technique for the management of large image collections. It reduces the file size of an image without degrading its quality to an unacceptable level [Figure 7]. Compression techniques belong to one of two classes: ${ }^{[3,19]}$

a. Lossless compression techniques ensure that the original image is exactly reconstructed from the compressed data. These techniques take advantage of redundancy in 


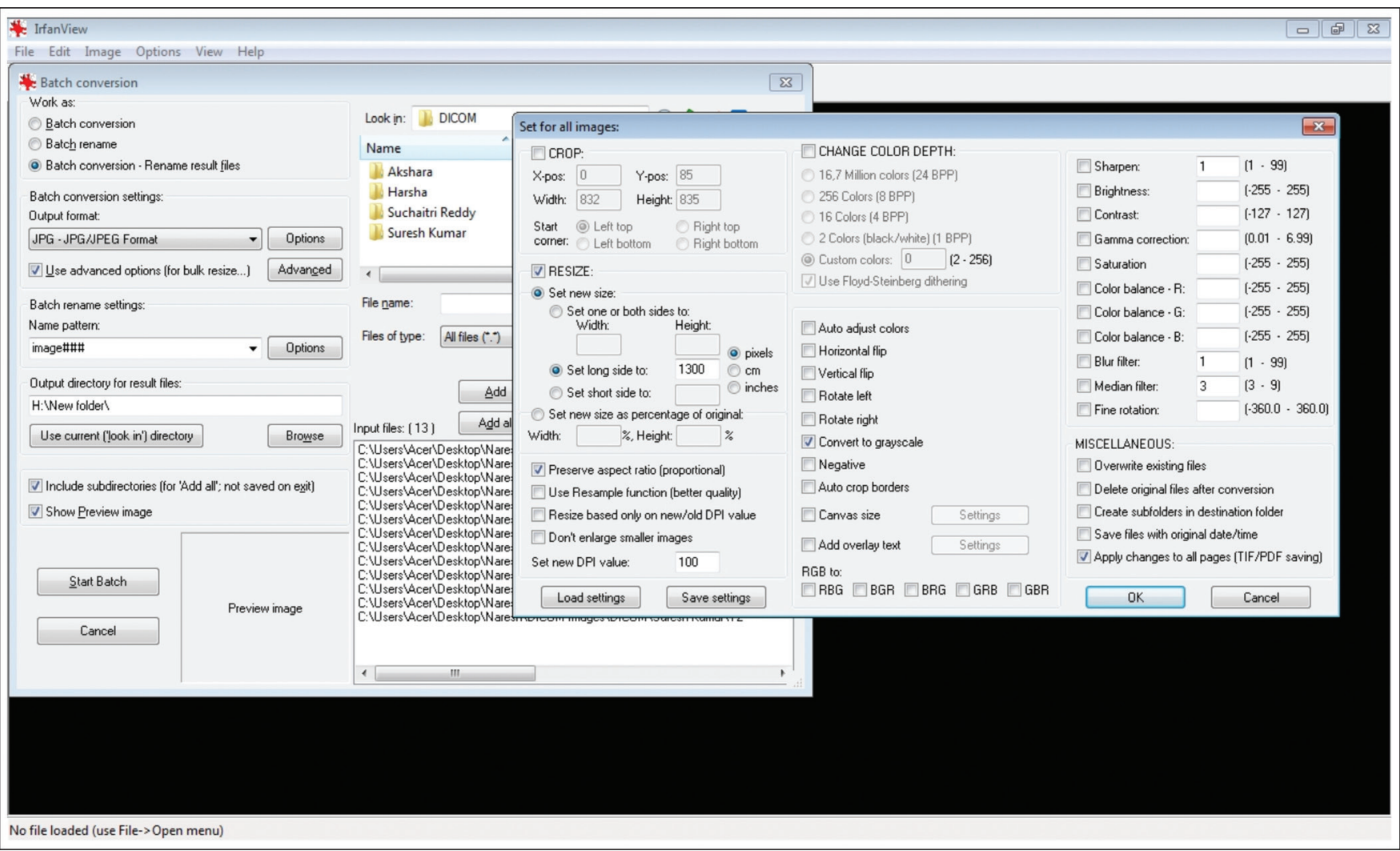

Figure 5: Screen saver from IrfanView (a popular image editing software package) performing batch operations. Note that batch operation such as conversion to different formats, renaming, cropping, resizing, and a host of image enhancements can be performed on a set of multiple images with a single click

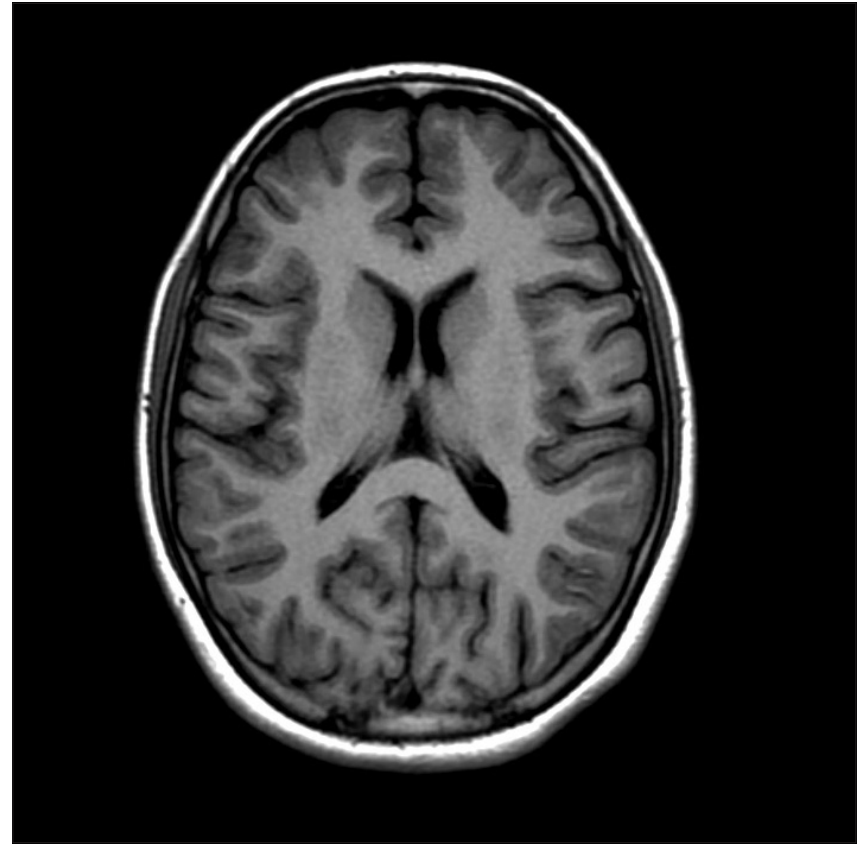

Figure 6: Axial T1W MRI of the brain. Though the entire image occupies hard disk space for storage, only the central part contains diagnostic information

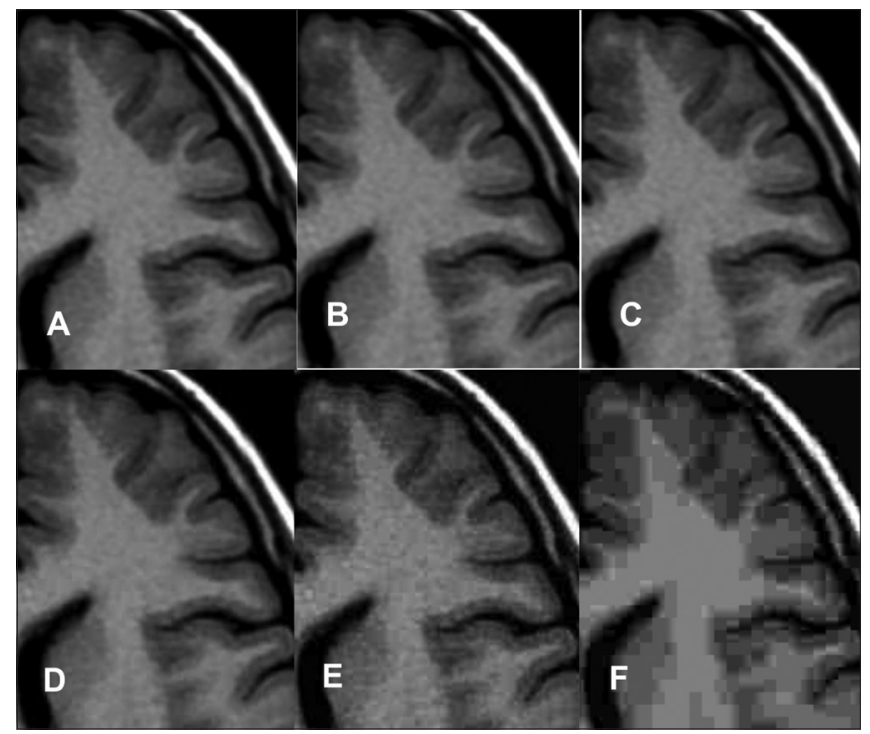

Figure 7 (A-F): Compression and image quality: axial T1W MRI of the brain saved as uncompressed TIFF image (A), TIFF image with lossless compression (B), uncompressed JPEG image (C), JPEG image with lossy compression (D) (reduction of image quality to $80 \%$ of original), JPEG image with lossy compression (E) (reduction of image quality to $40 \%$ of original) and JPEG image with lossy compression (F) (reduction of image quality to $10 \%$ of original). Note that for lossy compression techniques, increase in degree of compression results in an unacceptable loss of image quality 
data and eliminate it. They generally permit reduction of file sizes by a factor of 2 or 3 .

b. Lossy algorithms allow reconstruction of an approximation of the original data. Depending on the degree of compression, visually appreciable distortions may find their way into the image. These schemes often reduce the file sizes by a factor of 10 or more.

TIFF files usually employ lossless compression, while JPEG format may use either lossless or lossy compression schemes ${ }^{[3,13]}$ The advantage of using the JPEG format is that we can define the degree of compression depending on the anticipated utility of the image. Most image editing software packages permit image compression as a subroutine during batch processing of images.

\section{How can I Organize my Image Collection?}

Radiologists often archive images for later use at presentations or in publications. Due to the digital nature of the format, it is now very easy to resize, crop, sharpen, and annotate radiological images and perform other operations to achieve the best quality for presentation or publication. A challenge that the radiologist faces when preparing a presentation is in finding the best image among hundreds or thousands of images randomly archived in different folders. Often, it is necessary to browse through and view hundreds of images - with names like DSC 841.jpg or DSC 842.jpg - before one can find the right ones. To address this issue, the image collection has to archived, sorted, and organized efficiently, so that images can be found easily when required.

A first step when starting an image collection is to organize images into folders and subfolders [Figure 8]. For example, a neuroradiology image collection may be organized into separate folders according to the location of pathology, with each folder containing subfolders based on the type of pathology; the subfolders can, in turn, contain further subdivisions named after the actual pathological diagnosis. The final branch of this subfolder structure would contain the images pertinent to that diagnosis. This is a useful method to organize small image collections, especially when the diagnostic categories are relatively straight-forward.

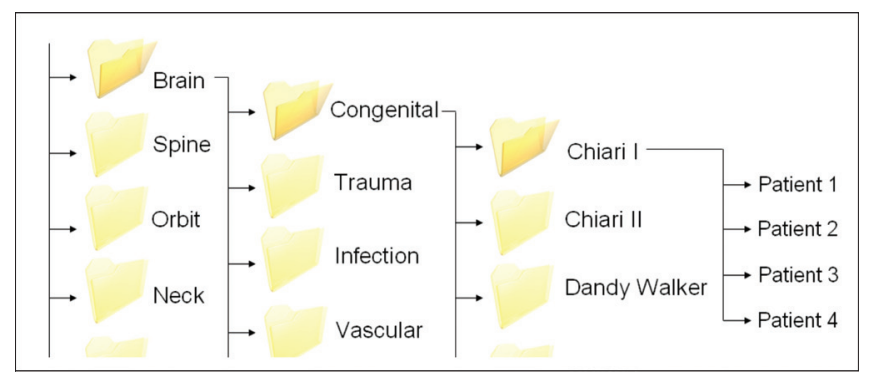

Figure 8: Organizing an image collection using hierarchical folders
Locating the correct image from such a collection is quick and intuitive.

Another method of organizing images is by renaming them so as to reflect the image content. In this technique, using a program that performs batch renaming of image files, all filenames with terms that contain diagnostic labels are appended and easily traced later using the "search" command of Windows ${ }^{\circledR}$ Explorer. The only problem with this technique is that the file names are often long.

An easier and more elegant way of organizing images is by using image "metadata." Earlier in this article, we have seen that all DICOM files have a header which is composed of several tags that contain information regarding the image. Images in other formats such as JPEG also have small bits of information - called metadata - written into the image file. EXIF tag structures and IPTC are the two types of metadata that are commonly employed to tag images. ${ }^{[20-22]}$ EXIF (exchangeable image file format) generally contains information regarding the date and time of creation of the image as well as the details of the camera used to create the image. Thus, if one wants to sort an image collection by date, a software package that can access the EXIF metadata is needed. IPTC stands for International Press Telecommunications Council, the organization that developed this standard. The IPTC Information Interchange Model is a file structure and set of metadata attributes that can be applied to text, images, and other media types. These attributes have gained near universal acceptance among photographers for embedding information into images. ${ }^{[23]}$

Photographers who have large collections of digital photographs use IPTC metadata to organize their collections. Typically, the photographer enters his name, contact information, captions, keywords, and copyright information to tag his images. Similarly, for radiology images, these fields are used to enter information such as clinical history, diagnosis, comments, and copyright information [Figure 9]. Once all the images are tagged, a search for specific images can be performed within an entire collection using the IPTC keywords. This is similar to searching images on the World Wide Web using Google Image search. Windows ${ }^{\circledast}$ Explorer and other software packages that are compatible with IPTC metadata can be used to perform such searches. A list of such software packages can be found at the IPTC website. ${ }^{[21]}$

\section{How can I Display a Stack of Images in PowerPoint ${ }^{\circledR}$ ?}

Microsoft ${ }^{\circledR}$ PowerPoint $^{\circledR}$ is a popular tool for on-screen presentations at national and international meetings. PowerPoint ${ }^{\circledR}$ presentations can contain text, images, figures, and animations. However, for radiologists, some limitations of PowerPoint ${ }^{\circledR}$ make it less than perfect in certain situations. 


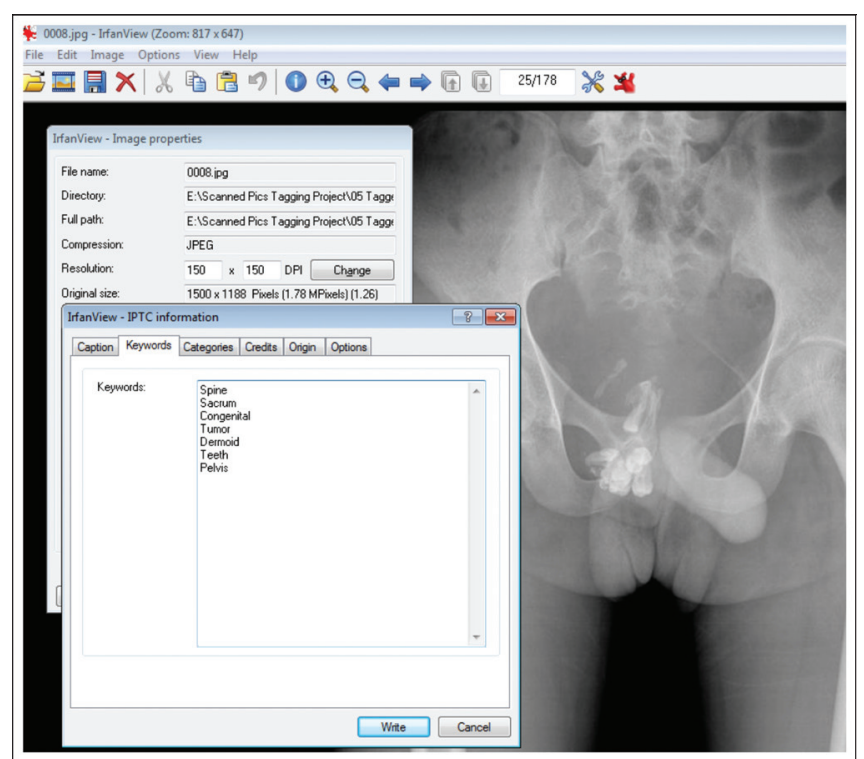

Figure 9: Display of IPTC information embedded in a JPEG image of a radiograph. The diagnosis of sacral dermoid has been categorized under the keywords "spine," "sacrum," "congenital," "tumor," "dermoid," "teeth," and "pelvis." Searching the image collection using any of the above keywords would result in display of this image

The true nature and extent of pathology may not always be apparent on a single image or few selected images that are displayed on a PowerPoint ${ }^{\circledR}$ slide. For example, displaying a series of axial images of the abdomen would be more effective in depicting the imaging findings in a case of abdominal tuberculosis than showing a few representative images. However, manually incorporating a series of images into a PowerPoint ${ }^{\circledast}$ presentation can be a time-consuming and tedious job.

Fortunately, there are several options for performing this with minimal effort. Software packages such as PACstacker, StackView, and RadViewer have been developed to enable import of a series of images and to display them interactively as a stack. ${ }^{[24-26]}$ While PACstacker and StackView operate as macros within PowerPoint ${ }^{\circledR}$, RadViewer is a Web-based software that converts images into a Flash file.

\section{How can I Display DICOM Files in my PowerPoint ${ }^{\circledR}$ Presentation?}

One other major drawback with PowerPoint ${ }^{\circledR}$ is that it does not provide support for DICOM images. Images need to be converted to other formats before they can be imported into the presentation. The resultant images will have a fixed contrast, set at the time of file conversion.

In order to address this problem, a plug-in called "Radfiler" has been created for PowerPoint ${ }^{\circledast}$. Incorporation of this plug-in gives the presenter the ability to directly import and display DICOM images. Further, it gives the presenter the freedom to scroll through image stacks, dynamically manipulate the window level and window width, and even to zoom and crop the images during the presentation. ${ }^{[27]}$

\section{How do I Create Presentations in a Short Time?}

There are certain situations where a large number of images have to be displayed in a presentation. For most users, using PowerPoint ${ }^{\circledR}$ for the job may be an overkill. Creating a presentation with a large set of digital images would take a while, as a separate slide has to be created for each photo. One other drawback with presentations in PowerPoint ${ }^{\circledR}$ is the lack of backward compatibility. It is frustrating to see hours of preparation go waste when a presentation saved in PowerPoint ${ }^{\circledR} 2007$ simply does not open on a computer that has an earlier version of PowerPoint ${ }^{\circledR}$ installed on it.

Fortunately, for rapid creation of slideshows, platformindependent software programs are available free of cost. Irfanview and Xnview are software programs that permit rapid creation of slideshows. ${ }^{[9,15]}$ It is possible to select a set of images, sort them, and convert them into a slideshow with just a few clicks. The slideshow can then be saved as an executable file that will run on any computer (irrespective of the operating system and the presence or absence of image management software) by simply double clicking the .exe file. The disadvantage with this mode of presentation is that it displays only one image per slide and it is not possible to mix images with text or other graphics.

\section{Conclusion}

This article introduces the reader to a few basic concepts regarding digital image management. It also outlines a variety of ways in which radiologists can make the most of the "digital" nature of these images, using tools that are freely available on the World Wide Web. There are several other such software programs and techniques that can be similarly used by radiologists to optimize their practice. It is essential to try out a number of such programs before finding the one that best suits each purpose.

\section{Acknowledgment}

The author extends his sincere thanks to Surg Capt IK Indrajit, whose ideas have played a major role in the content as well as final shape of this article.

\section{References}

1. Indrajit IK. Digital imaging and communications in medicine: A basic review. Indian J Radiol Imaging 2007;17:5-7.

2. DICOM. Available from: http://en.wikipedia.org/wiki/DICOM. [Last accessed on 2011 Jun 26].

3. Graham RN, Perriss RW, Scarsbrook AF. DICOM demystified: A 
review of digital file formats and their use in radiological practice. Clin Radiol 2005;60:1133-40.

4. Varma DR. Free DICOM browsers. Indian J Radiol Imaging 2008;18:12-6.

5. An introduction to the DICOM single-file format. Available from: http://www.cabiatl.com/mricro/dicom/index.html. [Last accessed on 2011 Jun 26].

6. Digital Imaging and Communications in Medicine (DICOM) Part 6: Data Dictionary. Available from: http://medical.nema.org/ dicom/2004/04_06PU.PDF. [Last accessed on 2011 Jun 26].

7. Dicomworks. Available from: http://dicom.online.fr/. [Last accessed on 2011 Jun 26].

8. ImageJ. Available from: http://rsbweb.nih.gov/ij/. [Last accessed on 2011 Jun 26].

9. Xnview. Available from: http://www.xnview.com/. [Last accessed on 2011 Jun 26].

10. FPImage. Available from: http://www.fpimage.com/info.html. [Last accessed on 2011 Jun 26].

11. Image file formats. Available from: http://en.wikipedia.org/wiki/ Image_file_formats. [Last accessed on 2011 Jun 26].

12. Ernst RD, Baumgartner BR, Tamm EP, Torres WE. Development of a Teaching File by Using a DICOM Database. Radiographics 2002;22:217-21.

13. Wiggins RH 2nd, Davidson HC, Harnsberger HR, Lauman JR, Geode PA. Image file formats: Past, present, and future. Radiographics 2001;21:789-98.

14. Medisp DICOM viewer. Available from: http://www.teiath.gr/ stef/tio/medisp/downloads.htm. [Last accessed on 2011 Jun 26].

15. Irfanview. Available from: http://www.irfanview.com/. [Last accessed on 2011 Jun 26].

16. Indrajit IK, Verma BS. Digital imaging in radiology practice: An introduction to few fundamental concepts. Indian J Radiol Imaging 2007;17:230-6.
17. Yam CS. Using PowerPoint to create high-resolution images for journal publications. AJR Am J Roentgenol 2005;185:273-6.

18. Stern EJ, Richardson ML. Preparation of digital images for presentation and publication. AJR Am J Roentgenol 2003;180: 1523-31.

19. 'File size, storage and image compression' in Digital Imaging for Photographers. In: Davies A, Fennessy P, editors. 4th ed. United Kingdom: Focal Press; 2001.p74 - 92.

20. Metadata. Available from: http://en.wikipedia.org/wiki/Metadata. [Last accessed on 2011 Jun 26].

21. International Press Telecommunications Council. Available from: http://www.iptc.org/site/Photo_Metadata/Overview/. [Last accessed on 2011 Jun 26].

22. Exchangeable image file format. Available from: http://en.wikipedia. org/wiki/Exif. [Last accessed on 2011 Jun 26].

23. IPTC Information Interchange Model. Available from: http:// en.wikipedia.org/wiki/IPTC_Information_Interchange_Model. [Last accessed on 2011 Jun 26].

24. Khanna PC, Thapa MM, de Regt D, Weinberger E. PACStacker: An enhancement of the scientific and educational capabilities of PowerPoint. AJR Am J Roentgenol 2009;192:W71-4.

25. Gniadek TJ, Desjardins B. Interactive display of stacks of images in scientific presentations with PowerPoint. AJR Am J Roentgenol 2004;183:859-61.

26. Park MY, Choi BG. What radiologists want to see: Stack mode display in PowerPoint. AJR Am J Roentgenol 2010;194:909-11.

27. Haider MA. Extending PowerPoint with DICOM image support. Radiographics 2003;23:1683-7.

Cite this article as: Varma DR. Managing DICOM images: Tips and tricks for the radiologist. Indian J Radiol Imaging 2012;22:4-13.

Source of Support: Nil, Conflict of Interest: None declared.

\section{Announcement}

\section{Android App}

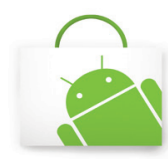

A free application to browse and search the journal's content is now available for Android based mobiles and devices. The application provides "Table of Contents" of the latest issues, which are stored on the device for future offline browsing. Internet connection is required to access the back issues and search facility. The application is compatible with all the versions of Android. The application can be downloaded from https://market.android.com/details?id=comm.app.medknow. For suggestions and comments do write back to us. 